\title{
Impact of Mindfulness-Based Cognitive Therapy on Intolerance of Uncertainty in Patients with Panic Disorder
}

\author{
Min Kuk Kim, Kang Soo Lee, Borah Kim, Tai Kiu Choi, and Sang-Hyuk Lee ${ }^{凶}$ \\ Department of Psychiatry, CHA Bundang Medical Center, CHA University, Seongnam, Republic of Korea
}

\begin{abstract}
Objective Intolerance of uncertainty (IU) is a transdiagnostic construct in various anxiety and depressive disorders. However, the relationship between IU and panic symptom severity is not yet fully understood. We examined the relationship between IU, panic, and depressive symptoms during mindfulness-based cognitive therapy (MBCT) in patients with panic disorder.

Methods We screened 83 patients with panic disorder and subsequently enrolled 69 of them in the present study. Patients participating in MBCT for panic disorder were evaluated at baseline and at 8 weeks using the Intolerance of Uncertainty Scale (IUS), Panic Disorder Severity Scale-Self Report (PDSS-SR), and Beck Depression Inventory (BDI).

Results There was a significant decrease in scores on the IUS ( $<<0.001)$, PDSS ( $<<0.001)$, and BDI ( $<<0.001)$ following MBCT for panic disorder. Pre-treatment IUS scores significantly correlated with pre-treatment PDSS $(\mathrm{p}=0.003)$ and BDI $(\mathrm{p}=0.003)$ scores. We also found a significant association between the reduction in IU and PDSS after controlling for the reduction in the BDI score ( $\mathrm{p}<0.001$ ).

Conclusion IU may play a critical role in the diagnosis and treatment of panic disorder. MBCT is effective in lowering IU in patients with panic disorder.

Psychiatry Investig 2016;13(2):196-202
\end{abstract}

Key Words Intolerance of uncertainty, Mindfulness-based cognitive therapy, Panic disorder.

\section{INTRODUCTION}

Intolerance of uncertainty (IU) refers to a "dispositional characteristic that results from a set of negative beliefs about uncertainty and its implications, and involves the tendency to react negatively on an emotional, cognitive, and behavioral level to uncertain situations and events." It was initially suggested that IU was specifically associated with excessive worry in generalized anxiety disorder. ${ }^{2-4}$ Recently, however, many studies suggested that IU may be a transdiagnostic construct in various anxiety and depressive disorders. ${ }^{5-9}$

Few studies have examined the relationship between IU and panic disorder. Sexton et al. ${ }^{4}$ reported that IU failed to predict panic symptom severity, as measured by the Beck Anxiety Inventory (BAI), in a non-clinical sample. In contrast, IU

Received: February 5, 2015 Revised: April 16, 2015

Accepted: May 4, 2015 Available online: March 10, 2016

$\triangle$ Correspondence: Sang-Hyuk Lee, MD, PhD

Department of Psychiatry, CHA Bundang Medical Center, CHA University, 59 Yatap-ro, Bundang-gu, Seongnam 463-712, Republic of Korea

Tel: +82-31-780-5694, Fax: +82-31-780-5862

E-mail: leesanghyuk@yahoo.com

(a) This is an Open Access article distributed under the terms of the Creative Commons Attribution Non-Commercial License (http://creativecommons.org/licenses/bync/3.0) which permits unrestricted non-commercial use, distribution, and reproduction in any medium, provided the original work is properly cited. significantly correlated with panic symptom severity as measured by the Body Sensations Questionnaire (BSQ) and Agoraphobic Cognitions Questionnaire (ACQ). ${ }^{7,10}$ Additionally, two studies reported that the Intolerance of Uncertainty Scale (IUS) score was significantly higher in patients with panic disorder than those in a non-clinical sample. ${ }^{8,11} \mathrm{~A}$ significant and substantial relationship between the IU inhibitory dimension and panic symptom severity was reported in a community sample. ${ }^{12}$ Similarly, in patients with panic disorder, there was a significant relationship between the IU inhibitory dimension and anxiety sensitivity (AS) somatic and cognitive dimensions with panic symptom severity. ${ }^{13}$

There are some reasons why researchers consider IU to be a potential panic disorder construct. First, patients with panic disorder frequently experience uncertain physical sensations, for which the cause and meaning are uncertain. Second, a panic attack itself accompanies a fair amount of uncertainty, as individuals cannot predict where and when a panic attack might occur and how long it might last. In this sense, individuals who can better tolerate this uncertainty may interpret this bodily sensation as less of a potential threat without catastrophizing. Therefore, they experience less frequent panic attacks. ${ }^{12}$ 
Mindfulness-based cognitive therapy (MBCT) was developed for depression relapse prevention. MBCT use subsequently expanded to treat various anxiety disorders, such as generalized anxiety disorder (GAD), post-traumatic stress disorder (PTSD), and panic disorder. ${ }^{14-16}$ Our group previously reported a reduction in panic symptom severity and AS following MBCT for panic disorder. To the best of our knowledge, no study has reported changes in IU during MBCT. ${ }^{17,18}$

Patients with panic disorder frequently suffer from depressive symptoms in addition to panic symptoms. ${ }^{19} \mathrm{MBCT}$ is an empirically supported treatment for both panic and depressive disorders. ${ }^{18,20}$ Therefore, we cannot exclude the possibility that panic symptom improvement is associated with improvement in depressive symptoms. In addition, panic patients exhibit various level of panic symptom prior to participating in MBCT. The level of baseline panic symptoms can be one factor influencing the reduction in panic symptom severity.

The present study was designed to investigate the relationship between IU, panic, and depressive symptoms during MBCT for panic disorder. Our hypotheses were as follows. First, IU, panic, and depressive symptoms would be significantly reduced in patients with panic disorder after MBCT. Second, the reduction in panic symptoms would be significantly correlated with pre-treatment panic symptom severity, the reduction in IU, and the reduction in depressive symptoms after MBCT. Third, the reduction in IU would significantly predict the reduction in panic symptom severity after controlling for pre-treatment panic symptom severity and the reduction in depressive symptoms after MBCT.

\section{METHODS}

\section{Subjects}

Subjects were recruited among panic disorder patients who were treated in the Department of Psychiatry, CHA Bundang Medical Center, from May 2012 to December 2013. After screening 83 patients, 69 patients were enrolled in the present study. Patients were enrolled in the present study if they were between 18 and 65 years of age and met the Diagnostic and Statistical Manual of Mental Disorders, Fourth Edition (DSMIV) criteria for panic disorder with or without agoraphobia following the structured clinical interview for DSM-IV (SCID), Axis I disorders (inter-rater reliability, k: 0.82). ${ }^{21,22}$ Experienced psychiatrists (SHL and BK) confirmed that the subjects were stable and that their remaining symptoms had not changed according to the Clinical Global Impressions (CGI) scale for at least 2 months prior to MBCT commencement.

Fourteen participants were excluded due to a history of comorbid Axis I disorders, mental retardation, personality disorders, or serious medical conditions (e.g., pregnancy, chronic obstructive pulmonary disease, bronchial asthma, cardiac disease, or thyroid disease). We excluded patients with comorbid psychiatric disorders in order to examine the relationship between IU, panic, and depressive symptoms in pure panic disorder during MBCT. Prior to the study, enrolled patients had not participated in other treatments, including psychotherapy, meditation, or yoga. Sixty-nine patients received MBCT for panic disorder. Among them, 27 failed to attend seven or more sessions, and were excluded from analysis. The flow of the current study is described in the Figure 1.

All patients took medication [mean \pm standard deviation (SD); $5.96 \pm 8.60$ months] prior to participating in the study. Medication used included: escitalopram (mean \pm SD; $10.83 \pm$ $4.69 \mathrm{mg} /$ day, 12 patients), paroxetine $(18.88 \pm 13.19 \mathrm{mg} /$ day, 29 patients), and sertraline ( $200.00 \mathrm{mg} /$ day, 1 patient) as antidepressants; and alprazolam $(0.56 \pm 0.30 \mathrm{mg} /$ day, 35 patients $)$ and clonazepam $(0.54 \pm 0.21 \mathrm{mg} /$ day, 19 patients $)$ as anxiolytics. Medications and dosages were not altered during the study. Using checklists, experienced clinicians monitored medication adherence of participants during individual interviews.

All study procedures complied with Bundang CHA Hospital Institutional Review Board regulations and all participants provided written informed consent.

\section{Assessments}

\section{IUS}

The 27-item IUS was used to assess intolerance of uncertainty in subjects who participated in MBCT for panic disorder. The IUS includes items that assess emotional, cognitive, and behavioral reactions to ambiguous situations, implications of being uncertain, and attempts to control the future (e.g., "It frustrates me not having all the information I need"). ${ }^{23}$ The items are rated using a 5-point Likert scale ranging from 1 ("Not at all characteristic of me") to 5 ("Entirely characteristic of me"). The IUS used in the present study was translated into Korean, and consisted of a 4-point Likert scale..$^{24}$ It had high internal consistency in both nonclinical and clinical samples. ${ }^{9,25}$ The internal consistency of the total score at pre-treatment in the current sample was high (Cronbach's alpha: 0.94).

\section{PDSS-SR}

The PDSS measures all panic disorder dimensions, including panic attack, anticipatory anxiety, panic-related phobias, wellness, severity of all symptoms, and impairment. ${ }^{26,27}$ The PDSS-SR is a 7-item self-report measure of panic disorder symptoms (e.g., "During the past week, how much have you worried or felt anxious about when your next panic attack would occur or about fears related to the attacks?"). ${ }^{28}$ Items are rated using a 5-point Likert scale ranging from 0 (none) to 


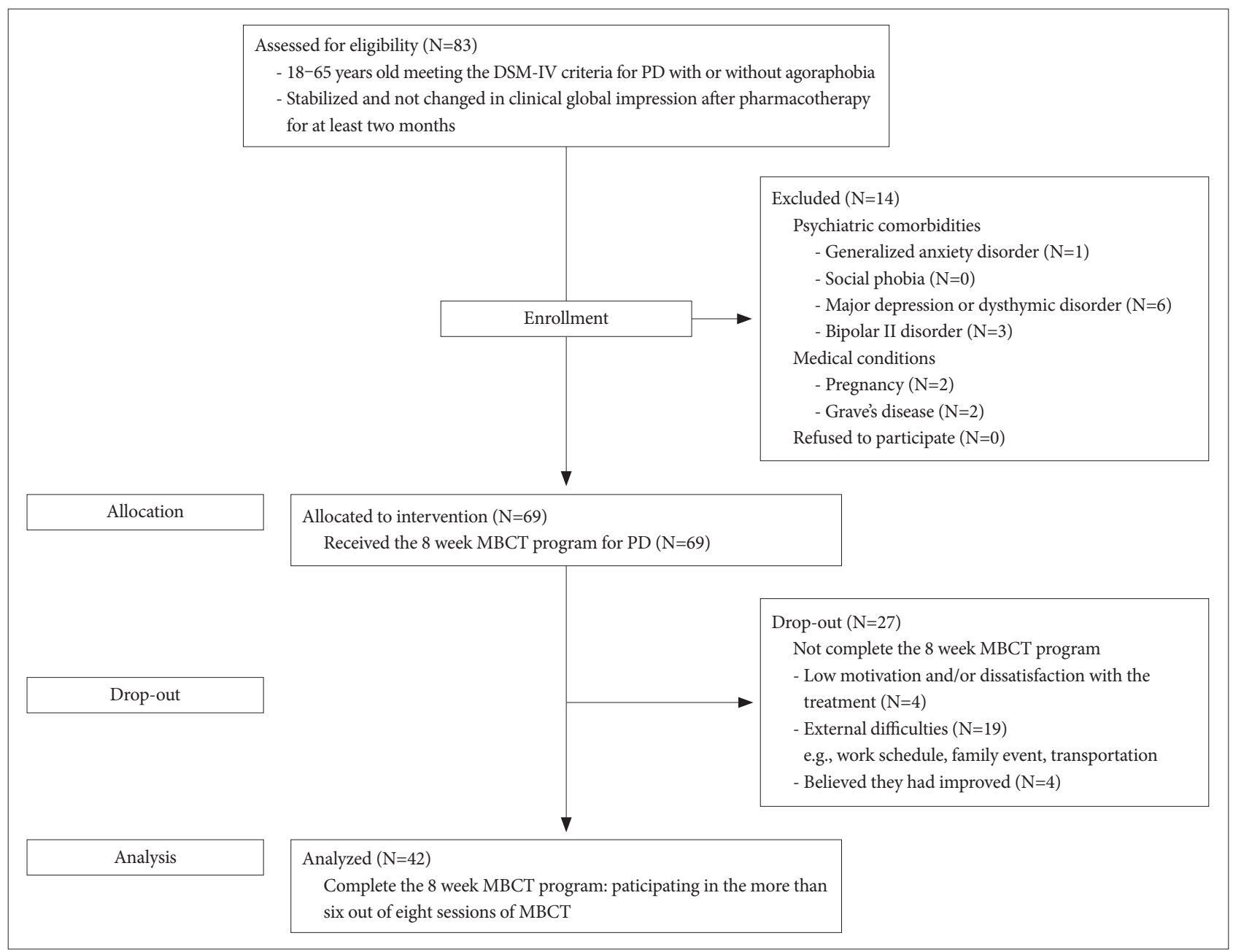

Figure 1. Flow diagram of the study of MBCT for panic disorder. MBCT: mindfulness-based cognitive therapy, PD: panic disorder.

4 (extreme). The Korean PDSS-SR showed good reliability and was satisfactory in a previous study. ${ }^{29}$ Internal consistency at pre-treatment was high in the current sample (Cronbach's alpha: 0.88).

\section{BDI}

The BDI was initially developed as a 21-item measure of depressive symptoms. In the present study, we used the Korean BDI (K-BDI), which has high internal consistency (Cronbach's alpha: 0.85 ) and discriminant validity. ${ }^{30-32}$ Internal consistency at pre-treatment was high in the current sample (Cronbach's alpha: 0.87).

\section{MBCT program}

The MBCT program used in the present study was led by three trained psychiatrists (SHL, SL, and MKK) who followed the MBCT manual for panic disorder. ${ }^{16,18}$ The program composed of 90 minute weekly sessions and homework for 8 weeks. The program was conducted in nine separate groups, each of which comprised up to eight participants. Weekly session components are described elsewhere. ${ }^{17}$ Briefly, the sessions included psychoeducation regarding causes, symptoms, cognitive model, and cognitive distortions that occur in panic disorder and mindfulness techniques (e.g., body scan, 3 min breathing space, sitting meditation, mindful walking, etc.). Homework was designed to encourage the participants to use mindfulness techniques in everyday life. Program adherence was monitored weekly using the homework checklist.

\section{Data analysis}

Sociodemographic characteristics were analyzed using the chi-square test and t-tests. Severity of panic symptoms, depression, and intolerance of uncertainty were analyzed over time using a repeated measures analysis of variance (ANOVA). Reductions in IUS, PDSS, and BDI scores following MBCT were calculated with the equation: [(pre-treatment score-post-treatment score)/pre-treatment score $\times 100(\%)]$. Correlation analysis was conducted to investigate the rela- 
tionship among pre-treatment PDSS score, the reduction in IU, BDI, and PDSS scores. Hierarchical regression analysis was then used to assess whether the reduction in IU could predict the reduction in the PDSS score after controlling for pretreatment PDSS score and the reduction in the BDI score, which were significant in the correlation analysis. SPSS 21.0 (IBM SPSS Statistics, Somers, New York, NY, USA) was used for statistical analysis. All reported probability values were two-tailed, with $\mathrm{p}<0.05$ considered statistically significant.

\section{RESULTS}

\section{Sociodemographic characteristics}

Sociodemographic characteristics of participants were as follows: age (mean $\pm \mathrm{SD}, 37.88 \pm 8.60$ years), gender [male/female $=15(36 \%) / 27(64 \%)]$, duration of illness $(4.71 \pm 5.01$ years), years of education $(14.63 \pm 2.18)$, marital status [married/unmarried $=33(79 \%) / 9(21 \%)]$, income status $[\geq \$ 5,850 /$ $\$ 3,900-5,850 /<\$ 3,900=13(31 \%) / 15(36 \%) / 14(33 \%)]$, and religion [religion declared/undeclared=23 (55\%)/19 (45\%)] (Table 1). Age, years of education, duration of illness, and economic status did not significantly correlate with the reduction in PDSS score following MBCT. There were no significant differences in PDSS reduction based on gender or marital status.

Table 1. Sociodemographic and Clinical Characteristics of participants who completed the MBCT Program $(\mathrm{N}=42)$

\begin{tabular}{lc}
\hline \multicolumn{1}{c}{ Variables } & Mean \pm SD or N (\%) \\
\hline Age (years) & $37.88 \pm 8.60$ \\
Gender, male/female & $15(36 \%) / 27(64 \%)$ \\
Duration of illness & $4.71 \pm 5.01$ \\
Education (years) & $14.63 \pm 2.18$ \\
Married/Unmarried & $33(79 \%) / 9(21 \%)$ \\
Income Status (\$/month) & \\
5,850 or more & $13(31 \%)$ \\
$3,900-5,850$ & $15(36 \%)$ \\
$\quad$ Less than 3,900 & $14(33 \%)$ \\
Religion declared/Undeclared & $23(55 \%) / 19(45 \%)$ \\
\hline
\end{tabular}

MBCT: mindfulness-based cognitive therapy, SD: standard deviation

\section{Changes in intolerance of uncertainty, panic and depressive symptom after MBCT}

Table 2 summarizes the changes in intolerance of uncertainty, panic, and depressive symptoms after MBCT. The repeated measures ANOVA revealed a significant decrease in IUS $(\mathrm{F}=11, \mathrm{df}=1, \mathrm{p}<0.001)$, PDSS $(\mathrm{F}=25, \mathrm{df}=1, \mathrm{p}<0.001)$, and BDI $(\mathrm{F}=73, \mathrm{df}=1, \mathrm{p}<0.001)$ after MBCT for panic disorder. Effect sizes (Cohen's d) were large for PDSS (0.82) and BDI (1.03), and moderate for IUS (0.51).

\section{Relationships between IU, panic symptom severity, and depression}

Figure 2 shows the relationship between IU, panic symptom severity, and depression. Pre-treatment IUS scores were significantly correlated with PDSS and BDI scores $(r=0.49$, $\mathrm{p}=0.003$ and $\mathrm{r}=0.51, \mathrm{p}=0.003$, respectively). Pre-treatment PDSS scores were significantly correlated with pre-treatment BDI scores $(r=0.62, \mathrm{p}=0.0001)$.

PDSS reduction was significantly correlated with IUS reduction and BDI reduction $(\mathrm{r}=0.54, \mathrm{p}=0.004$ and $\mathrm{r}=0.53$, $\mathrm{p}=0.002$, respectively). PDSS reduction was also significantly correlated with pre-treatment PDSS scores $(\mathrm{r}=0.41, \mathrm{p}=0.018)$.

\section{Hierarchical regression analyses predict the reduction in PDSS following MBCT}

Hierarchical regression analyses was performed to investigate whether intolerance of uncertainty could predict reductions in panic symptom severity following MBCT, after controlling for pre-treatment panic symptom severity and reductions in depressive symptoms. Table 3 summarizes the findings from the regression analyses. In the first step, the pretreatment PDSS score was entered. The model was statistically significant $[\mathrm{F}(1,49,850)=4.98, \mathrm{p}=0.004]$ and explained $17 \%$ of the variance in PDSS reduction. In step 2, the reduction in BDI was entered, and the model was still statistically significant $[\mathrm{F}(2,64006)=9.08, \mathrm{p}=0.001]$, and explained an additional $27 \%$ of the variance in PDSS reduction. Finally, IUS was entered in step 3 . The model was statistically significant $[\mathrm{F}$ (3, $53084)=8.92, \mathrm{p}<0.001]$ and explained an additional $11 \%$ of the variance in PDSS reduction after controlling for pre-treatment PDSS score and the BDI reduction following MBCT.

Table 2. Repeated measure ANOVA analyses on pre- and post-treatment PDSS, IUS, and BDI

\begin{tabular}{lcccccc}
\hline & $\begin{array}{c}\text { Pre-treatment } \\
(\text { mean } \pm \text { SD) }\end{array}$ & $\begin{array}{c}\text { Post-treatment } \\
(\text { mean } \pm \text { SD) }\end{array}$ & F & df & p & Effect size \\
\hline PDSS & $6.16 \pm 4.58$ & $3.00 \pm 2.98$ & 25 & 1 & $<0.001$ & 0.82 \\
IUS & $60.70 \pm 13.14$ & $53.52 \pm 15.21$ & 11 & 1 & $<0.001$ & 0.51 \\
BDI & $10.03 \pm 7.04$ & $3.95 \pm 4.44$ & 73 & 1 & $<0.001$ & 1.03 \\
\hline
\end{tabular}

Effect Size=Cohen's d. SD: standard deviation, PDSS: Panic Disorder Severity Scale, IUS: Intolerance of Uncertainty Scale, BDI: Beck Depression Inventory 

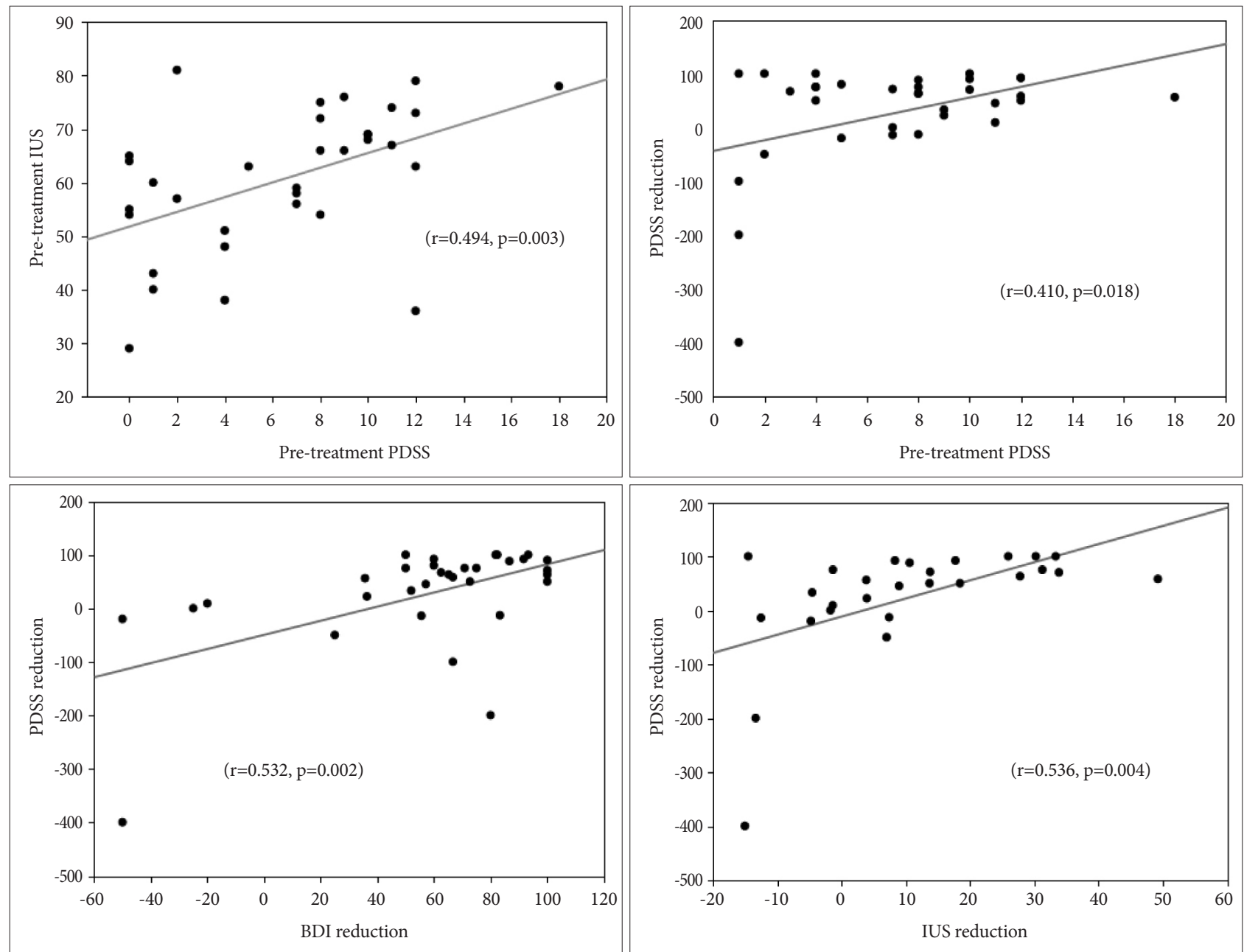

Figure 2. Relationships between variables. reduction (Pre-treatment score-Post-treatment score)/Pre-treatment score $\times 100 \%$. PDSS: Panic Disorder Symptom Severity, IUS: Intolerance of Uncertainty Scale, BDI: Beck Depression Inventory.

Table 3. Summary of hierarchical linear regression predicting the reductions in PDSS scores following MBCT

\begin{tabular}{lccccccc}
\hline & Predictors & $\Delta \mathrm{R} 2$ & $\mathrm{~B}$ & $\mathrm{SE}$ & $\mathrm{t}$ & $\mathrm{p}$ & $\mathrm{VIF}$ \\
\hline PDSS reduction & Step 1: Pre-treatment PDSS & $0.17^{*}$ & 10.43 & 4.67 & 2.23 & 0.035 & 1.000 \\
& Step 2: Pre-treatment PDSS & $0.27^{* *}$ & 9.29 & 3.94 & 2.36 & 0.027 & 1.008 \\
& BDI reduction & & 1.26 & 0.38 & 3.33 & 0.003 & 1.008 \\
& Step 3: Pre-treatment PDSS & $0.11^{* * *}$ & 9.69 & 3.62 & 2.68 & 0.014 & 1.010 \\
& BDI reduction & & 0.83 & 0.40 & 2.10 & 0.048 & 1.303 \\
& IUS reduction & & 2.43 & 1.06 & 2.29 & 0.032 & 1.293 \\
\hline
\end{tabular}

Reduction, [(pre-treatment score-post-treatment score)/pre-treatment score $\times 100$ (\%)], B represents the unstandardized regression coefficients for each step. ${ }^{*} \mathrm{p}<0.05,{ }^{* *} \mathrm{p}<0.01,{ }^{* * *} \mathrm{p}<0.001$. MBCT: mindfulness based cognitive therapy, PDSS: Panic Disorder Symptom Severity, BDI: Beck Depression Inventory, IUS: Intolerance of Uncertainty Scale

\section{DISCUSSION}

The present study demonstrated with statistical significance that IU, panic, and depressive symptoms in panic disorder patients can be substantially reduced by MBCT for panic disorder. In addition, IU was significantly correlated with panic disorder symptoms prior to treatment. Finally, the re- duction in IU was associated with the reduction in panic disorder symptoms after MBCT for panic disorder.

During MBCT for panic disorder, patients learn to be aware of their thoughts as an object to observe. Additionally, psychoeducation sessions teach patients to understand their common way of thinking, such as catastrophic misinterpretation. Lastly, by practicing attending to physical sensations in detail 
(e.g., breathing, touching, or moving) patients can better escape from further catastrophizing. In the face of uncertain bodily sensations (e.g., palpitation) or situations (e.g., riding public transportation), patients can become more aware and effectively handle catastrophizing misbeliefs using mindfulness techniques. Eventually, they can better tolerate those uncertainties.

This is the first intervention-based study to date that investigated the relationship between IU and panic symptom severity. Previous studies reported a significant reduction in IU in other anxiety disorders across treatments that were mostly based on cognitive behavioral therapy (CBT).$^{33-35}$ Both MBCT and CBT enhance one's ability to be aware of their thoughts; however, patient instructions regarding how misbeliefs should be handled significantly differ between the two techniques. While MBCT emphasizes acceptance of misbeliefs without judgment or elaboration, CBT encourages patients to correct misbeliefs using reevaluation of the belief and cognitive exposure. $^{33,36}$

We found a significant association among IU, panic, and depressive symptom severity prior to treatment. This result agrees with those of previous studies. ${ }^{12,13,37}$ In contrast, another group reported that IU was not significantly associated with panic disorder symptoms. ${ }^{4}$ This inconsistency may stem from the difference in sample selection and assessment measures used in previous studies. Only one study, by Carleton et al. ${ }^{13}$ was conducted in patients with panic disorder exclusively. ${ }^{13}$ Sexton et al. ${ }^{4}$ measured general anxiety symptom severity instead of panic symptom severity. ${ }^{4}$

The reduction in IU was significantly correlated with the reduction in panic symptoms after MBCT. This finding is in accordance with those of studies that reported a significant correlation between reductions in IU and anxiety symptoms in GAD, obsessive-compulsive disorder (OCD), and social phobia after CBT. ${ }^{33,34,36,38}$ In addition, greater reductions in IU were associated with greater reductions in panic symptoms after controlling for pre-treatment panic symptom severity and the reduction in depressive symptoms.

The present finding can be explained by the following mechanism. First, patients with panic disorder repeatedly experience a self-perpetuating cycle in which uncertain bodily sensations induce catastrophic misinterpretation, further anxiety, and more intense bodily sensations. It is presumed that enhancing patients' tolerance of uncertainty during MBCT for panic disorder might prevent this self-perpetuating cycle. Second, panic attack itself is an unpredictable and recurrent condition. Patients cannot predict when the next episode will be and how long it will last. Therefore, patients with panic disorder suffer from anticipatory anxiety (i.e., worry, fear, or concern that the attacks will occur again), and often develop avoidant behavior (e.g., not using public transportation or withdrawing from social activity). We postulate that patients learned to tolerate this inevitable uncertainty effectively due to $\mathrm{MBCT}$, and subsequently experience less anticipatory anxiety and avoidance symptoms.

Carleton et al. ${ }^{13}$ reported that IU and AS are independent vulnerabilities for panic disorder, and intervention may benefit from addressing both IU and AS. Although AS was not included in this study, our group previously reported a significant AS reduction after MBCT in patients with panic disorder. ${ }^{17,18}$ Accordingly, MBCT appears to be effective for patients with panic disorder.

The present study should be interpreted with caution for the following reasons. First, the small sample size of the study and the lack of a control group limited its statistical power and generalizability. Second, the drop out rate during MBCT in this study was relatively high. The more appropriate statistical method using intention to treat analysis may need to be considered in the future studies. Third, it is difficult to determine the causal relationship between IU, panic, and depressive symptom severity during MBCT. Experimental research that aims to determine the causal nature of the relationship may be necessary. Fourth, we did not use a scale to measure mindfulness proficiency, such as the Mindful Attention and Awareness Scale (MAAS), because a well-validated measure has not been developed in the Korean language.

In spite of the above limitations, the current findings support and extend those of previous studies suggesting that IU may be a panic disorder construct. ${ }^{12,13,37}$ The present study indicates that IU may have an important role in the diagnosis and treatment of panic disorder. Evidence exists suggesting that CBT reduces IU in various anxiety disorders; we now suggest that MBCT is an effective treatment option to lower IU in patients with panic disorder.

\section{Acknowledgments}

This research was supported in part by the National Research Foundation of Korea Basic Science Research Program funded by the Ministry of Education, Science, and Technology (2011-0023359) to S.H. Lee. Thanks to Sung-Jae Lee for conducting the MBCT program.

\section{REFERENCES}

1. Buhr K, Dugas MJ. The role of fear of anxiety and intolerance of uncertainty in worry: an experimental manipulation. Behav Res Ther 2009;47:215-223.

2. Dugas MJ, Gagnon F, Ladouceur R, Freeston MH. Generalized anxiety disorder: a preliminary test of a conceptual model. Behav Res Ther 1998;36:215-226.

3. van der Heiden C, Melchior K, Muris P, Bouwmeester S, Bos AE, van der Molen HT. A hierarchical model for the relationships between general and specific vulnerability factors and symptom levels of generalized anxiety disorder. J Anxiety Disord 2010;24:284-289.

4. Sexton KA, Norton PJ, Walker JR, Norton GR. Hierarchical model of 
generalized and specific vulnerabilities in anxiety. Cogn Behav Ther 2003;32:82-94.

5. Gentes EL, Ruscio AM. A meta-analysis of the relation of intolerance of uncertainty to symptoms of generalized anxiety disorder, major depressive disorder, and obsessive-compulsive disorder. Clin Psychol Rev 2011;31:923-933.

6. Starcevic V, Berle D. Cognitive specificity of anxiety disorders: a review of selected key constructs. Depress Anxiety 2006;23:51-61.

7. McEvoy PM, Mahoney AE. To be sure, to be sure: intolerance of uncertainty mediates symptoms of various anxiety disorders and depression. Behav Ther 2012;43:533-545.

8. Carleton RN, Mulvogue MK, Thibodeau MA, McCabe RE, Antony MM, Asmundson GJ. Increasingly certain about uncertainty: intolerance of uncertainty across anxiety and depression. J Anxiety Disord 2012;26:468-479.

9. Yook K, Kim KH, Suh SY, Lee KS. Intolerance of uncertainty, worry, and rumination in major depressive disorder and generalized anxiety disorder. J Anxiety Disord 2010;24:623-628.

10. McEvoy PM, Mahoney AE. Achieving certainty about the structure of intolerance of uncertainty in a treatment-seeking sample with anxiety and depression. J Anxiety Disord 2011;25:112-122.

11. Mahoney AE, McEvoy PM. A transdiagnostic examination of intolerance of uncertainty across anxiety and depressive disorders. Cogn Behav Ther 2012;41:212-222.

12. Carleton RN, Fetzner MG, Hackl JL, McEvoy P. Intolerance of uncertainty as a contributor to fear and avoidance symptoms of panic attacks. Cogn Behav Ther 2013;42:328-341.

13. Carleton RN, Duranceau S, Freeston MH, Boelen PA, McCabe RE, Antony MM. "But it might be a heart attack": intolerance of uncertainty and panic disorder symptoms. J Anxiety Disord 2014;28:463-470.

14. Evans S, Ferrando S, Findler M, Stowell C, Smart C, Haglin D. Mindfulness-based cognitive therapy for generalized anxiety disorder. J Anxiety Disord 2008;22:716-721.

15. Lynn SJ, Malakataris A, Condon L, Maxwell R, Cleere C. Post-traumatic stress disorder: cognitive hypnotherapy, mindfulness, and acceptance-based treatment approaches. Am J Clin Hypn 2012;54:311-330.

16. Kim YW, Lee SH, Choi TK, Suh SY, Kim B, Kim CM, et al. Effectiveness of mindfulness-based cognitive therapy as an adjuvant to pharmacotherapy in patients with panic disorder or generalized anxiety disorder. Depress Anxiety 2009;26:601-606.

17. Kim B, Cho SJ, Lee KS, Lee JY, Choe AY, Lee JE, et al. Factors associated with treatment outcomes in mindfulness-based cognitive therapy for panic disorder. Yonsei Med J 2013;54:1454-1462.

18. Kim B, Lee SH, Kim YW, Choi TK, Yook K, Suh SY, et al. Effectiveness of a mindfulness-based cognitive therapy program as an adjunct to pharmacotherapy in patients with panic disorder. J Anxiety Disord 2010;24:590-595.

19. Roy-Byrne PP, Stang P, Wittchen HU, Ustun B, Walters EE, Kessler RC. Lifetime panic-depression comorbidity in the National Comorbidity Survey. Association with symptoms, impairment, course and help-seeking. Br J Psychiatry 2000;176:229-235.

20. Piet J, Hougaard E. The effect of mindfulness-based cognitive therapy for prevention of relapse in recurrent major depressive disorder: a systematic review and meta-analysis. Clin Psychol Rev 2011;31:1032-1040.
21. Spitzer RL, Gibbon M, Williams JB. Structured Clinical Interview for Axis I DSM-IV Disorders (SCID). Washington, DC: American Psychiatric Association; 1995.

22. Han OS, Hong JP. Structured Clinical Interview for DSM-IV (SCID) Korean version. Seoul: Hana Medical; 2000.

23. Freeston MH, Rheaume J, Letarte H, Dugas MJ, Ladouceur R. Why do people worry? Pers Individ Differ 1994;17:791-802.

24. Choi HK. Dysfunctional Effects of Chronic Worry on Problem-Solving. Seoul: University of Joong-Ang, A Ph.D. dissertation; 1997.

25. Oh YA. The Relationship between Intolerance of Uncertainty and Worry: The Mediation Effects of Experiential Avoidance. Seoul: Catholic University; 2009.

26. Shear MK, Brown TA, Barlow DH, Money R, Sholomskas DE, Woods SW, et al. Multicenter collaborative panic disorder severity scale. Am J Psychiatry 1997;154:1571-1575.

27. Ballenger JC, Davidson JR, Lecrubier Y, Nutt DJ, Baldwin DS, den Boer JA, et al. Consensus statement on panic disorder from the International Consensus Group on Depression and Anxiety. J Clin Psychiatry 1998;59(Suppl 8):47-54.

28. Houck PR, Spiegel DA, Shear MK, Rucci P. Reliability of the self-report version of the panic disorder severity scale. Depress Anxiety 2002;15: 183-185.

29. Lee EH, Kim JH, Yu BH. Reliability and validity of the self-report version of the Panic Disorder Severity Scale in Korea. Depress Anxiety 2009;26:E120-E123.

30. Beck AT, Ward CH, Mendelson M, Mock J, Erbaugh J. An inventory for measuring depression. Arch Gen Psychiatry 1961;4:561-571.

31. Rhee MK, Lee YH, Park SH, Sohn CH, Chung YC, Hong SK, et al. A standardization study of Beck Depression Inventory (BDI): Korean version (K-BDI): reliability and factor analysis. Korean J Psychophathol 1995;4:77-95.

32. Rhee MK, Lee YH, Jung HY, Chio JH, Kim SH, Kim YK, et al. A Standardization Study of Beck Depression Inventory - Korean Version (KBDI) : Validity. Korean J Psychophathol 1995;4:96-104.

33. Dugas MJ, Ladouceur R. Treatment of GAD. Targeting intolerance of uncertainty in two types of worry. Behav Modif 2000;24:635-657.

34. Belloch A, Cabedo E, Carrio C, Fernandez-Alvarez H, Garcia F, Larsson C. Group versus individual cognitive treatment for ObsessiveCompulsive Disorder: changes in non-OCD symptoms and cognitions at post-treatment and one-year follow-up. Psychiatry Res 2011;187: 174-179.

35. Goldman N, Dugas MJ, Sexton KA, Gervais NJ. The impact of written exposure on worry: a preliminary investigation. Behav Modif 2007; 31:512-538.

36. Mahoney AE, McEvoy PM. Changes in intolerance of uncertainty during cognitive behavior group therapy for social phobia. J Behav Ther Exp Psychiatry 2012;43:849-854.

37. Mahoney AE, McEvoy PM. Trait versus situation-specific intolerance of uncertainty in a clinical sample with anxiety and depressive disorders. Cogn Behav Ther 2012;41:26-39.

38. Ladouceur R, Dugas MJ, Freeston MH, Leger E, Gagnon F, Thibodeau N. Efficacy of a cognitive-behavioral treatment for generalized anxiety disorder: evaluation in a controlled clinical trial. J Consult Clin Psychol 2000;68:957-964. 